\title{
Modelling soil salinity in Oued El Abid watershed, Morocco
}

\author{
El Mouatassime Sabri ${ }^{1 . *}$, Ahmed Boukdir ${ }^{2}$, Ismail Karaoui ${ }^{3}$, Abdelkrim Arioua ${ }^{4}$, Rachid \\ Messlouhi $^{5}$, Abdelkhalek El Amrani Idrissi ${ }^{6}$ \\ ${ }^{1}$ Sultan MoulaySlimane University, Department of Geology, Beni Mellal, Morocco \\ ${ }^{2}$ Sultan MoulaySlimane University, Department of Geology, Beni Mellal, Morocco \\ ${ }^{3}$ Sultan MoulaySlimane University, Department of Geology, Beni Mellal, Morocco \\ ${ }^{4}$ Sultan MoulaySlimane University, Department of Geology, Beni Mellal, Morocco \\ ${ }^{5}$ Agence de bassinhydraulique Oum Er Rbia|, Beni Mellal, Morocco \\ ${ }^{6}$ Agence de bassinhydraulique Oum Er Rbia, Beni Mellal, Morocco
}

\begin{abstract}
Soil salinisation is a phenomenon considered to be a real threat to natural resources in semi-arid climates. The phenomenon is controlled by soil (texture, depth, slope etc.), anthropogenic factors (drainage system, irrigation, crops types, etc.), and climate factors. This study was conducted in the watershed of Oued El Abid in the region of Beni Mellal-Khenifra, aimed at localising saline soil using remote sensing and a regression model. The spectral indices were extracted from Landsat imagery $(30 \mathrm{~m}$ resolution). A linear correlation of electrical conductivity, which was calculated based on soil samples (ECs), and the values extracted based on spectral bands showed a high accuracy with an $\mathrm{R}^{2}$ (Root square) of 0.80 . This study proposes a new spectral salinity index using Landsat bands B1 and B4. This hydro-chemical and statistical study, based on a yearlong survey, showed a moderate amount of salinity, which threatens dam water quality. The results present an improved ability to use remote sensing and regression model integration to detect soil salinity with high accuracy and low cost, and permit intervention at an early stage of salinisation.
\end{abstract}

\section{Introduction}

Soil salinity is a worldwide environmental problem [1] especially in arid and semi-arid climates [2]. Generally, it is calculated based on the electrical conductivity (EC) of the soil. In the last decade, many studies have aimed to identify the soil salinity using remote sensing based on satellite images [3]. In Morocco, as in many developing countries[4], the combination of poverty and population growth in fragile environments results the degradation of non-renewable resources. Salinity threatens about 500,000 hectares of land there, where most of it located upstream dams of water and belong to irrigated zones which treats natural resources as well as human livestock [5].

The phenomenon is controlled by several factors, including soil characteristics, climate factors, and anthropogenic factors, which make the control of the phenomenon difficult [6]. The sodium-rich soils have a $\mathrm{pH}$ of more than 8.2 with a preponderance of sodium carbonate and bicarbonate. This results in sodium saturation of the clay fraction, and leads

Corresponding author: e.sabri@usms.ma. 
to a compact and impermeable soil, which prevents aeration and penetration of water necessary for normal plant growth[7,8].

The watershed of Oued El Abid has a moderate rate of soil erosion, which is related to soil salinity [9]. Erosion, in turn, influences the spatial distribution of salinity, for example causing high salinity in the deposit area farther down the watershed, caused by inundation event. Furthermore, the accumulated water in the Bin El Ouidane dam, which is the main sources of irrigation the region, threatens agricultural lands downstream [10]. After irrigation, the water added to the soil is used by the crop or evaporates directly from the soil. The salt, however, is left behind in the soil and, if not removed, by (calcium sulfate dihydrate $(\mathrm{CaSO} 4 * 2 \mathrm{H} 2 \mathrm{O}))$, accumulates. This negatively impacts the limited agricultural land, reduces production, and often leads to land being abandoned, leaving a bare surface with occasional shrubs [11].

This study covers the watershed of Oued El Abid with an area of $7686 \mathrm{~km}^{2}$ in the upper part of the immense OumEr-Rbia watershed $\left(50,000 \mathrm{~km}^{2}\right)$ in the region of TadlaAzilal between the High Atlas and the plain of Tadla. The main water course is the Oued El Abid, a standout amongst the most essential water assets of Morocco, which aliment with the Oued Assif Ahensal the Bin El Ouidane dam, utilized for water system and hydropower (Fig. 1).

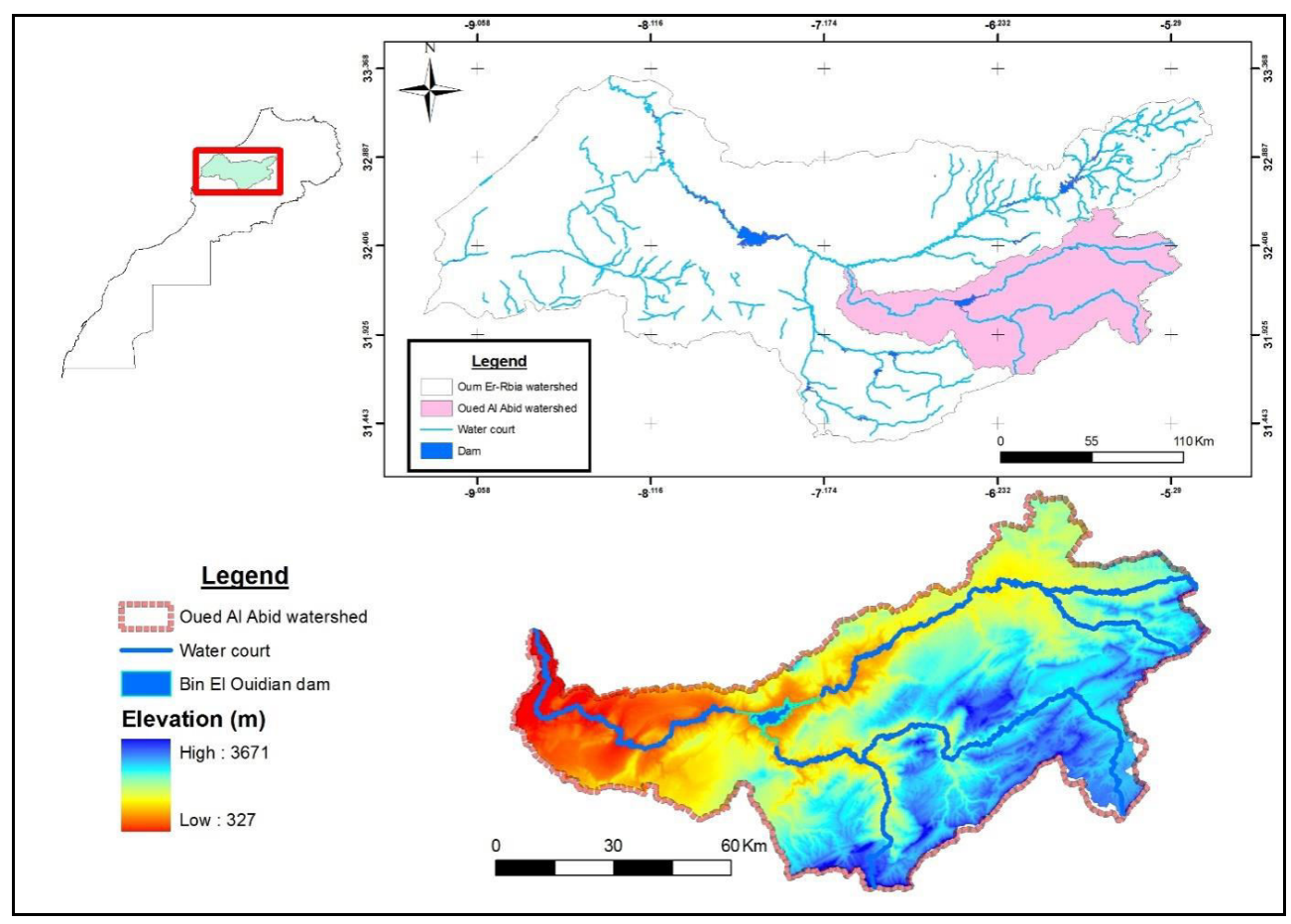

Fig. 1. Study area

The overall aim of this study was to develop effective combined spectral-based statistical regression models using Landsat images to introduce an approach to map salinity hazard, the application of which should help track down the occurrence of salt in the subsoil at an early stage. 


\section{Methodology}

To localize the affected regions in the watershed, an approach using an integration of remote sensing and regression model was used (Fig. 2). The usefulness of remote sensing has been improved by many studies, in particular those based on visible spectral bands [12].

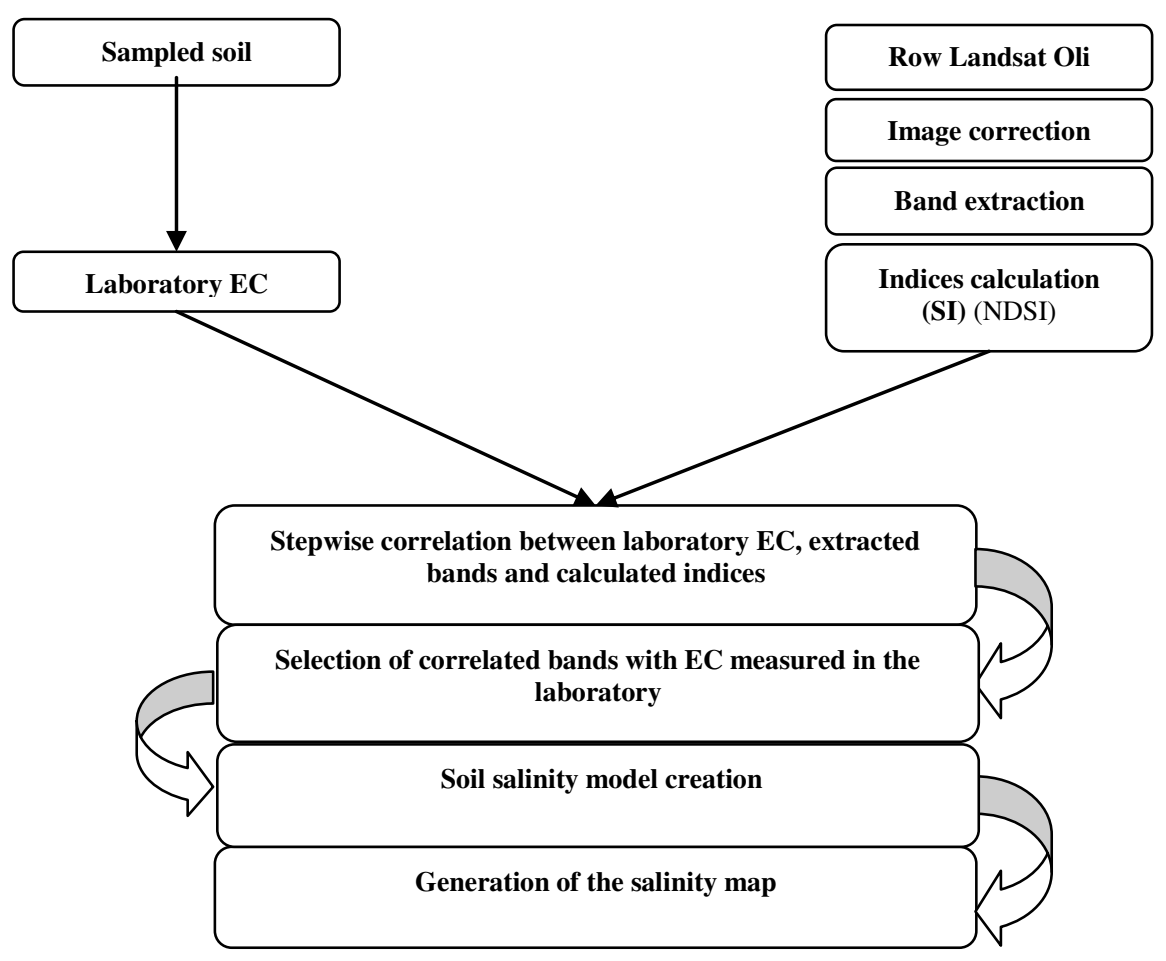

Fig. 2. Methodology

The EC was calculated in the laboratory based on saturated paste extracts from soil samples (Tab. 1) collected in the field [12].

Table 1. Bands data of soil samples.

\begin{tabular}{|c|c|c|c|c|c|c|c|c|c|c|c|c|c|c|}
\hline Point & $x$ & $Y$ & Z & EC & B1 & B2 & B3 & B4 & B5 & B6 & B7 & NDSI & SI & Panchro \\
\hline units & $\mathrm{m}$ & $\mathrm{m}$ & $\mathrm{m}$ & $\mu \mathrm{S} / \mathrm{cm}$ & - & - & - & - & - & - & - & - & - & - \\
\hline P 1 & 345637 & 184470 & 263 & 533 & 0.18 & 0.18 & 0.2 & 0.26 & 0.35 & 0.41 & 0.37 & $\begin{array}{c}- \\
0.13\end{array}$ & 0.19 & 0.24 \\
\hline P 2 & 344284 & 189718 & 266 & 578 & 0.12 & 0.1 & 0.09 & 0.05 & 0.52 & 0.15 & 0.07 & 0.27 & 0.1 & 0.07 \\
\hline P 3 & 346298 & 173082 & 285 & 589 & 0.17 & 0.16 & 0.18 & 0.24 & 0.32 & 0.37 & 0.34 & $\begin{array}{c}- \\
0.15 \\
\end{array}$ & 0.18 & 0.22 \\
\hline P 4 & 368941 & 159905 & 560 & 640 & 0.12 & 0.1 & 0.07 & 0.07 & 0.18 & 0.13 & 0.09 & 0.05 & 0.09 & 0.07 \\
\hline P 5 & 390459 & 170007 & 670 & 731 & 0.11 & 0.08 & 0.05 & 0.04 & 0.08 & 0.05 & 0.03 & 0.14 & 0.08 & 0.06 \\
\hline P 6 & 399180 & 167559 & 845 & 752 & 0.14 & 0.13 & 0.13 & 0.15 & 0.29 & 0.31 & 0.25 & $\begin{array}{c}- \\
0.07\end{array}$ & 0.14 & 0.11 \\
\hline P 7 & 411017 & 171767 & 915 & 805 & 0.12 & 0.1 & 0.09 & 0.12 & 0.16 & 0.2 & 0.18 & $\begin{array}{c}- \\
0.14\end{array}$ & 0.1 & 0.1 \\
\hline P 8 & 413438 & 177963 & 1433 & 845 & 0.16 & 0.16 & 0.19 & 0.26 & 0.36 & 0.42 & 0.36 & $\begin{array}{c}- \\
0.15\end{array}$ & 0.17 & 0.22 \\
\hline
\end{tabular}




\begin{tabular}{|c|c|c|c|c|c|c|c|c|c|c|c|c|c|c|}
\hline P 9 & 426061 & 179763 & 909 & 851 & 0.12 & 0.1 & 0.07 & 0.08 & 0.1 & 0.08 & 0.07 & $\begin{array}{c}- \\
0.05\end{array}$ & 0.09 & 0.07 \\
\hline P 10 & 419074 & 182648 & 1416 & 892 & 0.1 & 0.08 & 0.06 & 0.05 & 0.06 & 0.06 & 0.05 & 0.07 & 0.08 & 0.04 \\
\hline P 11 & 428600 & 181453 & 1098 & 933 & 0.1 & 0.08 & 0.05 & 0.03 & 0.07 & \begin{tabular}{|l|}
0.03 \\
\end{tabular} & 0.02 & 0.2 & \begin{tabular}{|l|} 
\\
\end{tabular} & 0.04 \\
\hline P 12 & 440598 & 189712 & 1114 & 946 & 0.15 & 0.13 & 0.14 & 0.16 & 0.2 & 0.24 & 0.23 & 0.06 & 0.14 & 0.13 \\
\hline P 13 & 448196 & $\mid 191857$ & 1404 & 983 & 0.11 & 0.09 & 0.09 & 0.12 & 0.2 & 0.26 & 0.23 & $\begin{array}{c}- \\
0.15\end{array}$ & 0.1 & 0.08 \\
\hline P 14 & 460365 & 194792 & 1555 & 993 & 0.13 & 0.11 & 0.12 & 0.13 & 0.28 & 0.3 & 0.22 & 0.06 & 0.12 & 0.11 \\
\hline P 15 & 473463 & 193341 & 1530 & 1000 & 0.15 & 0.14 & 0.14 & 0.16 & 0.24 & 0.26 & 0.23 & 0.06 & 0.14 & 0.16 \\
\hline P 16 & 477042 & 203032 & 1562 & 1017 & 0.12 & 0.11 & 0.11 & 0.15 & 0.21 & 0.29 & 0.25 & 0.15 & 0.12 & 0.13 \\
\hline P 17 & 474299 & 200025 & 1384 & 1104 & 0.11 & 0.09 & 0.07 & 0.07 & 0.12 & 0.1 & 0.08 & 0.02 & 0.09 & 0.09 \\
\hline P 18 & 473671 & 207147 & 1535 & 1109 & 0.12 & 0.1 & 0.09 & 0.1 & 0.19 & 0.16 & 0.13 & -0.1 & 0.1 & 0.08 \\
\hline P 19 & 463220 & 207420 & 1454 & 1143 & 0.13 & 0.11 & 0.11 & 0.13 & 0.21 & 0.23 & 0.19 & 0.09 & 0.12 & 0.11 \\
\hline P 20 & 410229 & 164435 & 1211 & 1148 & 0.13 & 0.11 & 0.12 & 0.17 & 0.23 & 0.28 & 0.25 & 0.17 & 0.12 & 0.12 \\
\hline P 21 & 423739 & $\mid 159237$ & 1137 & 1155 & 0.13 & 0.12 & 0.12 & 0.13 & 0.28 & 0.23 & 0.17 & $\begin{array}{c}- \\
0.04 \\
\end{array}$ & 0.12 & 0.13 \\
\hline P 22 & 428461 & 155976 & 1151 & 1167 & 0.13 & 0.12 & 0.11 & 0.11 & 0.17 & 0.18 & \begin{tabular}{|l|} 
\\
\end{tabular} & 0.02 & 0.12 & 0.1 \\
\hline P 23 & 434055 & 147979 & 1367 & 1183 & 0.12 & 0.11 & 0.11 & 0.13 & 0.23 & 0.25 & 0.21 & $\begin{array}{c}- \\
0.07 \\
\end{array}$ & 0.12 & 0.1 \\
\hline P 24 & 435443 & 153220 & 1734 & 1184 & 0.12 & 0.11 & 0.11 & 0.14 & 0.32 & 0.28 & 0.21 & \begin{tabular}{|c|}
- \\
0.11 \\
\end{tabular} & 0.12 & 0.13 \\
\hline P 25 & 438287 & 164126 & 1294 & 1215 & 0.15 & 0.15 & 0.2 & 0.29 & 0.41 & 0.51 & 0.43 & $\begin{array}{c}- \\
0.19\end{array}$ & 0.17 & 0.22 \\
\hline P 26 & 448972 & 163708 & 1486 & 1216 & 0.16 & 0.16 & 0.18 & 0.23 & 0.32 & 0.37 & 0.32 & 0.12 & 0.17 & 0.18 \\
\hline P 27 & 418806 & 185929 & 1707 & 1267 & 0.17 & 0.17 & 0.19 & 0.23 & 0.28 & 0.31 & 0.26 & $\begin{array}{c}- \\
0.09 \\
\end{array}$ & 0.18 & 0.21 \\
\hline P 28 & 434579 & 187102 & 1409 & 1287 & 0.12 & 0.12 & 0.12 & 0.17 & 0.26 & 0.34 & 0.28 & 0.19 & 0.12 & 0.13 \\
\hline P 29 & 440235 & 196160 & 1600 & 1338 & 0.12 & 0.11 & 0.11 & 0.15 & 0.19 & 0.29 & 0.24 & \begin{tabular}{|c}
- \\
0.13 \\
\end{tabular} & 0.12 & 0.09 \\
\hline P 30 & 444117 & 208698 & 1052 & 1383 & 0.14 & 0.13 & 0.14 & 0.18 & 0.34 & 0.37 & 0.28 & 0.12 & 0.14 & 0.1 \\
\hline P 31 & 452653 & 207356 & 1322 & 1508 & 0.14 & 0.13 & 0.15 & 0.25 & 0.37 & 0.49 & 0.4 & $\begin{array}{c}- \\
0.25 \\
\end{array}$ & 0.15 & 0.19 \\
\hline P 32 & 467816 & 199214 & 1420 & 1563 & 0.11 & 0.09 & \begin{tabular}{|l|}
0.08 \\
\end{tabular} & 0.08 & 0.16 & 0.15 & 0.1 & 0 & 0.1 & 0.06 \\
\hline P 33 & 476825 & 183970 & 1752 & 1580 & 0.12 & 0.1 & 0.08 & 0.07 & 0.08 & 0.08 & 0.07 & 0.05 & 0.09 & 0.06 \\
\hline P 34 & 469864 & 182667 & 2118 & 1639 & 0.13 & 0.11 & \begin{tabular}{|l|}
0.12 \\
\end{tabular} & 0.14 & 0.21 & 0.25 & 0.21 & \begin{tabular}{|l|}
-0.1 \\
\end{tabular} & 0.12 & 0.13 \\
\hline P 35 & 462673 & 179683 & 2079 & 1643 & 0.12 & 0.11 & 0.13 & 0.17 & $\mid$\begin{tabular}{|l|}
0.24 \\
\end{tabular} & 0.35 & 0.31 & \begin{tabular}{|c|}
- \\
0.15 \\
\end{tabular} & 0.12 & 0.14 \\
\hline P 36 & 454659 & 176501 & 2154 & 1756 & 0.14 & 0.13 & 0.14 & 0.16 & 0.19 & 0.26 & 0.23 & $\begin{array}{c}- \\
0.07\end{array}$ & 0.14 & 0.14 \\
\hline P 37 & 448580 & 174642 & 2362 & 1846 & 0.12 & 0.11 & 0.11 & 0.13 & 0.16 & 0.2 & 0.18 & $\begin{array}{c}- \\
0.07\end{array}$ & 0.12 & 0.12 \\
\hline P 38 & 438156 & 185927 & 1434 & 2805 & 0.12 & 0.11 & 0.1 & 0.1 & 0.16 & 0.16 & 0.13 & 0.04 & 0.11 & 0.09 \\
\hline P 39 & 396756 & 167243 & 1222 & 2917 & 0.13 & 0.12 & 0.11 & 0.12 & 0.2 & 0.22 & 0.18 & $\begin{array}{c}- \\
0.05 \\
\end{array}$ & 0.12 & 0.11 \\
\hline
\end{tabular}




\begin{tabular}{|c|c|c|c|c|c|c|c|c|c|c|c|c|c|c|}
\hline P 40 & 389967 & 157998 & 1367 & 3080 & 0.13 & 0.11 & 0.12 & 0.14 & 0.23 & 0.28 & 0.23 & $\begin{array}{c}- \\
0.09\end{array}$ & 0.12 & 0.11 \\
\hline P 41 & 394236 & 150337 & 1765 & 3300 & 0.1 & 0.07 & 0.04 & 0.03 & 0.06 & \begin{tabular}{|l|}
0.03 \\
\end{tabular} & 0.01 & 0.24 & 0.06 & 0.04 \\
\hline P 42 & 410169 & 131201 & 2223 & 5240 & 0.09 & 0.07 & 0.05 & 0.04 & 0.04 & \begin{tabular}{|l|}
0.04 \\
\end{tabular} & 0.03 & 0.09 & 0.07 & 0.04 \\
\hline P 43 & 430213 & 137871 & 2025 & 5440 & 0.13 & 0.13 & 0.15 & 0.19 & 0.29 & \begin{tabular}{|l|} 
\\
\end{tabular} & 0.3 & 0.13 & 0.14 & 0.15 \\
\hline
\end{tabular}

To obtain accurate analytical results, each of the 43 soil samples was reported based on the mean of five subsamples taken within a $1600 \mathrm{~m}^{2}$ area. The mean sample was attributed to the central sample. Each subsample consists of about $1 \mathrm{~kg}$ of top soil taken at $30 \mathrm{~cm}$ depth. The samples' ECs ranged from 533 to $5440 \mu \mathrm{s} / \mathrm{cm}$, with a mean salinity value equal to $1454 \mu \mathrm{s} / \mathrm{cm}$.

Remote sensing was used to extract indices based on Landsat 8 Oli images from the same date as the field sampling. Those images are captured by the Landsat sensor launched on February 11, 2013, which consists of two science instruments, the Operational Land Imager (OLI) and the Thermal Infrared Sensor (TIRS). These two sensors composed from 11 bands (Tab.2) provide seasonal coverage of the global landmass at a spatial resolution of 30 meters (visible, NIR, SWIR), 100 meters (thermal), and 15 meters (panchromatic) [13].

Table 2. Landsat 8 Band specifications, Bands 1 to 9 are Oli spectral bands while bands 10-11 are TIRS spectral bands.

\begin{tabular}{|l|c|c|}
\hline Spectral Band & Wavelength (um) & Resolution(m) \\
\hline Band 1- Coastal/ Aerosol & $0.433-0.453$ & 30 \\
\hline Band 2- Blue & $0.450-0.515$ & 30 \\
\hline Band 3- Green & $0.525-0.600$ & 30 \\
\hline Band 4- Red & $0.630-0.680$ & 30 \\
\hline Band 5- Near Infrared (NIR) & $0.845-0.885$ & 30 \\
\hline Band 6- Short Wavelength Infrared (SWIR) & $1.560-1.600$ & 30 \\
\hline Band 7- Short Wavelength Infrared (SWIR) & $2.100-2.300$ & 30 \\
\hline Band 8- Panchromatic & $0.500-0.680$ & 15 \\
\hline Band 9- Cirrus & $1.360-1.390$ & 30 \\
\hline Band 10-Long Wavelength Infrared & $10.30-11.30$ & 100 \\
\hline Band 11- Long Wavelength Infrared & $11.50-12.50$ & 100 \\
\hline
\end{tabular}

The images were used after a radiometric- atmospheric treatments and classification to obtain high-accuracy results using ENVI, the radiometric correction consists of conversion from the sensor measurement to a physical quantity, when the sensor is measuring a radiance from the top of the atmosphere, the radiance is normalized into a reflectance values. Where the Dark Object Subtraction (DOS) has used for atmospheric correction to calibrate the luminance measured in the image. The map layout and indices extraction were done in ArcGIS software.

The validation of the obtained values from Landsat images by comparison to the soil sample analysis was done using a regression model in JMP13 [15].

\section{Results}

The results show a high correlation between the Landsat Oli image bands (B1 and B4) and EC with an acceptable $\mathrm{R}^{2}(0.80)$ and root-mean-square error $(\mathrm{RMSE}=488.56)$ (Tab. $3)$. 
Table 3. Results of regression model

\begin{tabular}{|c|c|c|c|c|c|c|c|c|c|}
\hline \multicolumn{2}{|c|}{$\begin{array}{l}\text { Squares Deviations } \\
\text { Sum (SDS) }\end{array}$} & $\begin{array}{c}\text { freedom } \\
\text { Degree of } \\
\text { error (FDE) }\end{array}$ & RMSE & $\begin{array}{c}\mathbf{R} \\
\text { square }\end{array}$ & $\begin{array}{l}\text { R square } \\
\text { adjusted }\end{array}$ & Cp & $\mathbf{p}$ & AlCc & BIC \\
\hline \multicolumn{2}{|c|}{9547841.4} & 40 & 488.5653 & 0.8024 & 0.7926 & 15.23675 & 3 & 660.4382 & 666.4304 \\
\hline \multicolumn{10}{|c|}{ Curent estimation } \\
\hline Lock & Grasped & Coefficient & Estimation & \multicolumn{2}{|c|}{$\begin{array}{c}\text { Freedom } \\
\text { Degrees of } \\
\text { the } \\
\text { numerator }\end{array}$} & \multicolumn{2}{|c|}{ Squares Sum } & $\begin{array}{l}\text { "Report } \\
\text { F" }\end{array}$ & "Prob.>F" \\
\hline \multirow{11}{*}{$\checkmark$} & & Constant & -4794.054 & & 1 & 0 & & 0 & 1 \\
\hline & & B5 & 0 & & 1 & 672122.5 & & 2.953 & 0.09363 \\
\hline & & NDSI & 0 & & 1 & 1061142 & & 4.876 & 0.03317 \\
\hline & & SI & 0 & & 1 & 105193.7 & & 0.434 & 0.51368 \\
\hline & & B6 & 0 & & 1 & 2184699 & & 11.572 & 0.00156 \\
\hline & & B7 & 0 & & 1 & 2815671 & & 16.311 & 0.00024 \\
\hline & & Panch & 0 & & 1 & 382144.8 & & 1.626 & 0.2098 \\
\hline & & B1 & 48583.234 & & 1 & 9463436 & & 39.646 & $1.81 \mathrm{E}-07$ \\
\hline & & B2 & 0 & & 1 & 171716.3 & & 0.714 & 0.40319 \\
\hline & & B3 & 0 & & 1 & 22891.78 & & 0.094 & 0.76112 \\
\hline & & B4 & 281.30091 & & 1 & 3748.665 & & 0.016 & 0.9009 \\
\hline
\end{tabular}

Based on these results, a model (Eq. 1) was integrated with Landsat imagery to produce a soil salinity map (Fig. 3).

$$
E C=48583.23 *(\text { Band } 1)+281.30 *(\text { Band } 4)-4794.05
$$




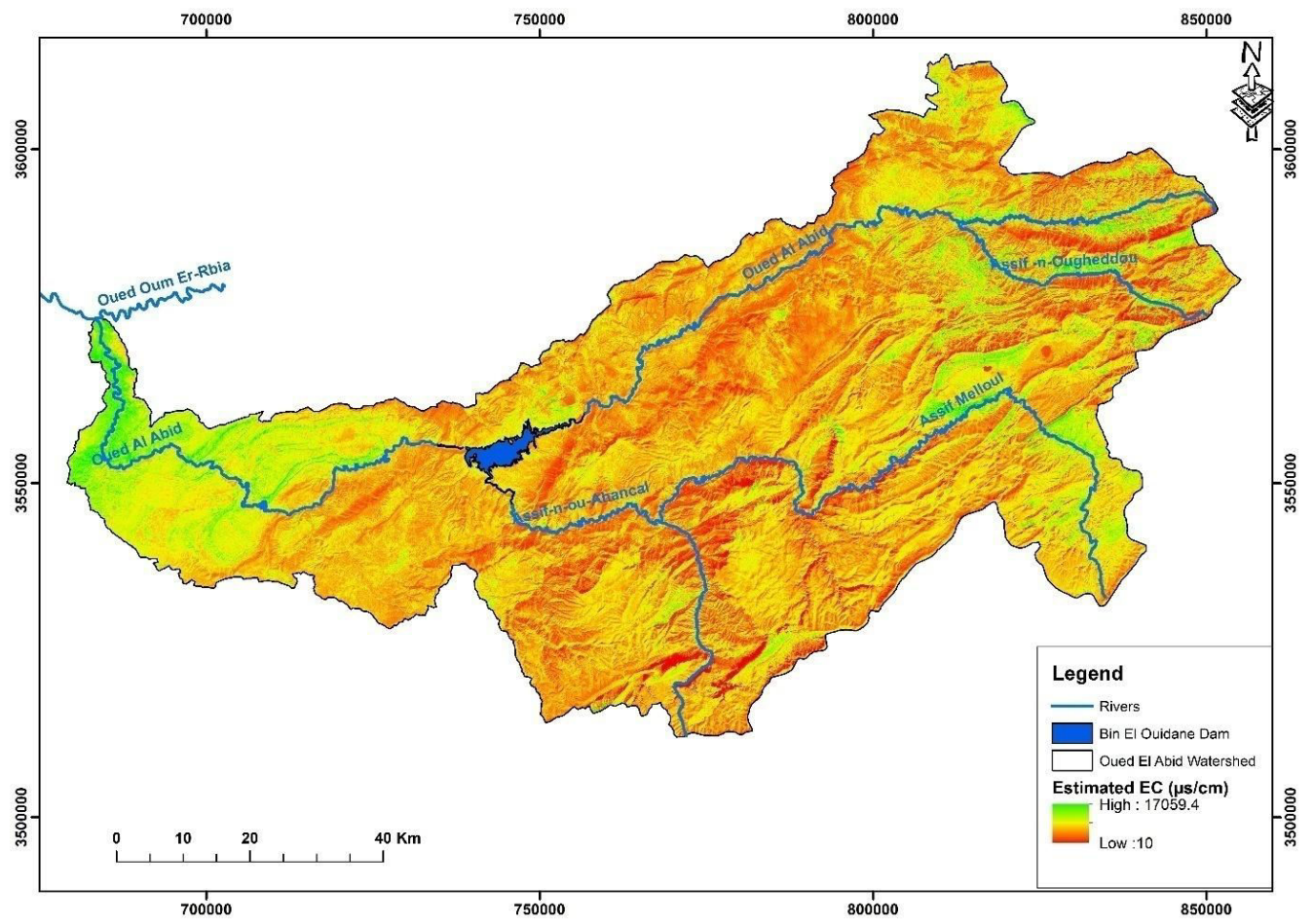

Fig. 3. Soil salinity map

A survey was done to compare the measurements obtained from the model and the salinity measured in field in order to validate the model results, as shown in the table below (Tab. 4).

Table 4. Measurement validation in $\mu \mathrm{s} / \mathrm{cm}$

\begin{tabular}{|c|c|c|c|c|c|c|c|c|}
\hline Sample & units & \multicolumn{1}{|c|}{ P1 } & P2 & P3 & P4 & P5 & P6 & P7 \\
\hline $\mathrm{X}$ & $\mathrm{m}$ & 348075.9 & 375184.9 & $\begin{array}{l}438346 . \\
0\end{array}$ & 414870.2 & 444773.9 & 470485.5 & 481943.9 \\
\hline $\mathrm{Y}$ & $\mathrm{m}$ & 170743.4 & 159564.5 & $\begin{array}{l}183878 \\
7\end{array}$ & 150062.4 & 183040.3 & 203441.9 & 176053.4 \\
\hline $\mathrm{EC} \mathrm{M}$ & $\mu \mathrm{S} / \mathrm{cm}$ & 2800.67 & 2025.27 & 733 & 413.67 & 1249.02 & 1583.88 & 1280.54 \\
\hline EC E & $\mu \mathrm{S} / \mathrm{cm}$ & 2820.86 & 2017.19 & 721.84 & 414.42 & 1256.95 & 1590.21 & 1286.31 \\
\hline
\end{tabular}

EC M: Electrical conductivity measured

EC E: Electrical conductivity estimated

\section{Discussion}

The main contribution of this study is the use of remote sensing to localize area affected by salinisation. Our results prove the capability of the methodology to identify affected areas and show a high correlation between laboratory analyses and the model presented 
The acceptable $\mathrm{R}^{2}$ demonstrates the usefulness of Landsat satellite imagery even at medium resolutions - high resolution is not necessary to get an accurate result.

The map shows moderate and distributed salinity upstream of Bin El Ouidane dam, where soil erosion occurs at high rates [9] and increases downstream of the dam, which explains the agricultural lands affected by saline irrigation water $[16,17,18]$.

The survey (Tab. 4) shows similarity of result of measured and model estimation with a standard deviation of $10 \mu \mathrm{s} / \mathrm{cm}$.

Indeed, the obtained results has its limits by lack of historical datasets and unpublished studies concern the study area which could be helpful to validate accurately the model, adding some limits related to survey area as the topography which restrict our mobility and limits the spatial distribution of soil samples, the things which could affect the accuracy of the results and validity of the model.

\section{Conclusion}

This study shows the ability of remote sensing to map soil salinity by coupling satellite image bands to a regression model. With the simplicity of the model and accuracy of the result, remote sensing becomes a reliable source to map soil salinity and could provide important support to decision makers and planners to simulate scenarios for the evolution of salinity and plan interventions against it. This study also demonstrates that even a relatively low satellite image resolution of $30 \mathrm{~m}$ can yield a highly accurate map of soil salinity, which may be especially useful in large study areas where high image resolution is an obstacle.

\section{References:}

1. A. Allbed, L. Kumar, Adv. Remote Sens. 2, 373-385 (2013)

2. K. Nagaz, F. E. Mokh, M. M. Masmoudi, N. B. Mechlia, JAEID. 111, 207-223 (2017)

3. G.I. Metternicht, J.A. Zinck, Remote Sens. Environ. 85, 1-20 (2003)

4. P. Blaikie, The political economy of soil erosion in developing countries. Third World Planning Rev. 7, 356 (2016)

5. K.F. Benbrahim, M. Ismaili, S.F. Benbrahim, A. Tribak, Sci. Chang. Planétaires Sécheresse. 15, 307-320 (2004)

6. J. Wu, P. Li, H. Qian, Y. Fang, Environ. Earth Sci. 71, 3465-3475 (2014)

7. A. Khalil, L. Hanich, A. Bannari, L. Zouhri, O. Pourret, R. Hakkou, J. Geochem. Explor. 125, 117-129(2013)

8. A.Bannari, G. Kadhem, A. El-Battay, N.A. Hameid, M. Rouai, Adv. Remote Sens. 5, 315 (2016)

9. E.M. Sabri, A. Boukdir, M. Rachid, A.E.L.Mahboul, V.M. Romaric, A. Zitouni, Z. Echakraoui, Agrofor Int. J. 2, 99-108 (2017)

10. H. Ouatiki, A. Boudhar, Y. Tramblay, L. Jarlan, T. Benabdelouhab, L. Hanich, M.R. El Meslouhi, A. Chehbouni, Climate. 5, 1(2017)

11. M.N. Qadir, A.D. Karajeh, B. F George, IWMI. 5, 29 (2015)

12. R.E. Ontiveros, L. Diakite-Diakite, M.E. Álvarez-Sánchez, P.M. Coras-Merino, Tecnol. Cienc. Agua. 4, 127-140 (2013)

13. S.Nawar, H. Buddenbaum, J. Hill, J. Kozak, Remote Sens. 6, 10813-10834 (2014) 
14. T. Dube, O. Mutanga, ISPRS J. Photogramm. Remote Sens. 101, 36-46 (2015)

15. R. Carver, SAS Institute. Second edition (2014)

16. M. Barbouchi, R. Abdelfattah, K. Chokmani, N. Ben aissa, R. Lhissou, A. El Harti, IEEE J. Sel. Top. Appl. Earth Obs. Remote Sens. 8, 3823-3832 (2015)

17. A. El Harti, R. Lhissou, K. Chokmani, J. Ouzemou, M. Hassouna, E.M. Bachaoui, A. El Ghmari, Int. J. Appl. Earth Obs. Geoinformation. 50, 64-73( 2016)

18. I. Karaoui, A. Arioua, A. El Amrani Idrissi, W. Nouaim, D. Elhamdouni, K.A. Ouhamchich, M. Hssaisoune, R. Hnini, J. Geosci. Environ. Prot. 5, 121-132(2017) 